\title{
A DISCRETE FIXED POINT THEOREM OF EILENBERG AS A PARTICULAR CASE OF THE CONTRACTION PRINCIPLE
}

\author{
JACEK JACHYMSKI
}

Received 6 November 2003

We show that a discrete fixed point theorem of Eilenberg is equivalent to the restriction of the contraction principle to the class of non-Archimedean bounded metric spaces. We also give a simple extension of Eilenberg's theorem which yields the contraction principle.

\section{Introduction}

The following theorem (see, e.g., Dugundji and Granas [2, Exercise 6.7, pages 17-18]) was presented by Samuel Eilenberg on his lecture at the University of Southern California, Los Angeles in 1978. (I owe this information to Professor Andrzej Granas.) This result is a discrete analog of the Banach contraction principle (BCP) and it has applications in automata theory.

Theorem 1.1 (Eilenberg). Let $X$ be an abstract set and let $\left(R_{n}\right)_{n=0}^{\infty}$ be a sequence of equivalence relations in $X$ such that

(i) $X \times X=R_{0} \supseteq R_{1} \supseteq \cdots$;

(ii) $\bigcap_{n=0}^{\infty} R_{n}=\Delta$, the diagonal in $X \times X$;

(iii) given a sequence $\left(x_{n}\right)_{n=0}^{\infty}$ such that $\left(x_{n}, x_{n+1}\right) \in R_{n}$ for all $n \in \mathbb{N}_{0}$, there is an $x \in X$ such that $\left(x_{n}, x\right) \in R_{n}$ for all $n \in \mathbb{N}_{0}$.

If $F$ is a self-map of $X$ such that given $n \in \mathbb{N}_{0}$ and $x, y \in X$,

$$
(x, y) \in R_{n} \Longrightarrow(F x, F y) \in R_{n+1},
$$

then $F$ has a unique fixed point $x_{*}$ and $\left(F^{n} x, x_{*}\right) \in R_{n}$ for each $x \in X$ and $n \in \mathbb{N}_{0}$.

(The letter $\mathbb{N}_{0}$ denotes the set of all nonnegative integers.) A direct proof of Theorem 1.1 will be given in Section 2. However, our main purpose is to show that Eilenberg's theorem (ET) is equivalent to the restriction of BCP to the class of non-Archimedean bounded metric spaces. This will be done in Section 3. Recall that a metric $d$ on a set $X$ is called non-Archimedean or an ultrametric (see de Groot [1] or Engelking [3, page 504]) if

$$
d(x, y) \leq \max \{d(x, z), d(z, y)\} \quad \forall x, y, z \in X .
$$


Then, in fact, $d(x, y)=\max \{d(x, z), d(z, y)\}$ if $d(x, z) \neq d(z, y)$, and therefore, each nonArchimedean metric space has the extraordinary geometric property that each three points of it are vertices of an isosceles triangle. We notice that non-Archimedean metrics are useful tools in many problems of fixed point theory (see, e.g., [6, proofs of Theorems 3,4 , and 5] on connections between some nonlinear contractive conditions, [7,8] on converses to contraction theorems, [5, Example 1] concerning a comparison of two fixed point theorems of the Meir-Keeler type). Moreover, there is also a variant of the BCP for self-maps of a non-Archimedean metric space proved by Prieß-Crampe [10] (also see Petalas and Vidalis [9]).

It turns out that, in general, the contraction principle cannot be derived from ET since each mapping satisfying assumptions of the latter theorem need not be surjective unless its domain is a singleton (cf. Corollary 3.3). Therefore, in Section 4 we establish a slight extension of ET (cf. Theorem 4.1) which is strong enough to yield the contraction principle. A paper of Grudziński [4] goes in a similar direction; however, he was able to obtain only some particular cases of the contraction principle via a discrete argument. Finally, we discuss a variant of ET given by Rus [11] (cf. Remarks 2.1 and 4.3).

\section{Proof of Eilenberg's theorem}

We give here a direct proof of Theorem 1.1. Fix an $x \in X$. By (i), $(x, F x) \in R_{0}$. Hence by (1.1), we may infer that $\left(F^{n} x, F^{n+1} x\right) \in R_{n}$ for all $n \in \mathbb{N}_{0}$. By (iii), we obtain the existence of an $x_{*} \in X$ such that

$$
\left(F^{n} x, x_{*}\right) \in R_{n} \quad \forall n \in \mathbb{N}_{0} .
$$

Hence, again by (1.1), we get that $\left(F^{n+1} x, F x_{*}\right) \in R_{n+1}$; equivalently, $\left(F^{n} x, F x_{*}\right) \in R_{n}$ for all $n \in \mathbb{N}$, the set of all positive integers, and also for $n=0$ since $R_{0}=X \times X$. Thus, given $n \in \mathbb{N}_{0}$, we have by the symmetry that $\left(F x_{*}, F^{n} x\right) \in R_{n}$. Hence, by (2.1), we get using the transitivity that

$$
\left(F x_{*}, x_{*}\right) \in R_{n} \quad \forall n \in \mathbb{N}_{0} .
$$

By (ii), this yields that $x_{*}=F x_{*}$. We show that $x_{*}$ is a unique fixed point of $F$. Let $y_{*}=$ $F y_{*}$. Then $\left(x_{*}, y_{*}\right) \in R_{0}$, so by $(1.1),\left(F^{n} x_{*}, F^{n} y_{*}\right) \in R_{n}$, that is, $\left(x_{*}, y_{*}\right) \in R_{n}$ for all $n \in \mathbb{N}_{0}$. Hence by (ii), we get that $x_{*}=y_{*}$ which completes the proof.

Remark 2.1. Observe that a point $x$ from condition (iii) is uniquely determined: if $\left(x_{n}, x^{\prime}\right)$ $\in R_{n}$, then $\left(x^{\prime}, x_{n}\right) \in R_{n}$ and by the transitivity, $\left(x^{\prime}, x\right) \in R_{n}$ for all $n \in \mathbb{N}_{0}$ which, by (ii), yields that $x^{\prime}=x$. Actually, a minor modification of the above proof shows that the assumptions of Theorem 1.1 could be weakened: the relations $R_{n}$ need not be transitive if we assume the uniqueness of a point $x$ in condition (iii). This exactly was done in [11, Theorem 5].

\section{Eilenberg's theorem and the contraction principle}

Theorem 3.1. Let $F$ be a self-map of an abstract set $X$ and $\alpha \in(0,1)$. The following statements are equivalent. 
(i) There exists a sequence $\left(R_{n}\right)_{n=0}^{\infty}$ of equivalence relations in $X$ such that the assumptions of ET are satisfied.

(ii) There exists a non-Archimedean bounded and complete metric $d$ such that $F$ is an $\alpha$-contraction with respect to $d$.

Proof. (i) $\Rightarrow$ (ii). Given two distinct elements $x, y \in X$, it follows from Theorem 1.1(i) and (ii) that the set $\left\{n \in \mathbb{N}_{0}:(x, y) \in R_{n}\right\}$ is of a form $\{0,1, \ldots, p\}$ for some $p \in \mathbb{N}_{0}$. Then set $p(x, y):=p$. If $x=y$, set $p(x, y):=\infty$. Next define

$$
d(x, y):=\alpha^{p(x, y)} \quad \forall x, y \in X
$$

under the convention that $\alpha^{\infty}=0$. Clearly, $d(x, y)=0$ if and only if $x=y$. Since the function $p(\cdot, \cdot)$ is symmetric, so is $d$. We show that condition (1.2) holds. Fix elements $x, y, z$ in $X$. Without loss of generality, we may assume that $x, y$, and $z$ are distinct. Then $(x, z) \in R_{p(x, z)}$ and $(z, y) \in R_{p(z, y)}$. Set

$$
k:=\min \{p(x, z), p(z, y)\} .
$$

Since $\left(R_{n}\right)_{n=0}^{\infty}$ is descending, we get that $(x, z),(z, y) \in R_{k}$. By the transitivity, $(x, y) \in R_{k}$ and hence $p(x, y) \geq k$. Then, by the definition of $k$,

$$
d(x, y)=\alpha^{p(x, y)} \leq \alpha^{k}=\max \left\{\alpha^{p(x, z)}, \alpha^{p(z, y)}\right\}=\max \{d(x, z), d(z, y)\}
$$

which means that $d$ is a non-Archimedean metric. Clearly, $d$ is bounded. We show that $d$ is complete. Let $\left(x_{n}\right)_{n=1}^{\infty}$ be a Cauchy sequence in $(X, d)$. Then there is a subsequence $\left(x_{k_{n}}\right)_{n=0}^{\infty}$ such that

$$
d\left(x_{k_{n}}, x_{k_{n+1}}\right)<\alpha^{n} .
$$

Set $y_{n}:=x_{k_{n}}$. Since $d\left(y_{n}, y_{n+1}\right)<\alpha^{n}$, we may infer that $p\left(y_{n}, y_{n+1}\right)>n$. Hence and by the definition of $p,\left(y_{n}, y_{n+1}\right) \in R_{n}$. By hypothesis, there is a $y \in X$ such that $\left(y_{n}, y\right) \in R_{n}$ for all $n \in \mathbb{N}_{0}$. Then $p\left(y_{n}, y\right) \geq n$ which yields that $d\left(y_{n}, y\right) \leq \alpha^{n}$. Hence $\left(x_{n}\right)_{n=1}^{\infty}$ is convergent as a Cauchy sequence containing a convergent subsequence.

Finally, we show that $F$ is an $\alpha$-contraction. Fix two distinct elements $x, y \in X$. Then $(x, y) \in R_{p(x, y)}$, so by $(1.1),(F x, F y) \in R_{p(x, y)+1}$. Hence $p(F x, F y) \geq p(x, y)+1$ which implies that

$$
d(F x, F y)=\alpha^{p(F x, F y)} \leq \alpha^{p(x, y)+1}=\alpha d(x, y) .
$$

(ii) $\Rightarrow($ i). Define

$$
R_{n}:=\left\{(x, y) \in X \times X: d(x, y) \leq \alpha^{n} \delta(X)\right\} \quad \forall n \in \mathbb{N}_{0},
$$

where $\delta(X)$ denotes the diameter of $X$. Then it is obvious that $R_{n}$ are reflexive, symmetric and conditions (i) and (ii) of Theorem 1.1 hold. The transitivity of $R_{n}$ follows easily from (1.2). We verify condition (iii) of Theorem 1.1 . Assume that a sequence $\left(x_{n}\right)_{n=0}^{\infty}$ is such that

$$
\left(x_{n}, x_{n+1}\right) \in R_{n}
$$


for all $n \in \mathbb{N}_{0}$. Hence the series $\sum_{n=1}^{\infty} d\left(x_{n}, x_{n+1}\right)$ is convergent which implies that $\left(x_{n}\right)_{n=0}^{\infty}$ is a Cauchy sequence. By completeness, $x_{n} \rightarrow x$ for some $x \in X$. We prove that $\left(x_{n}, x\right) \in R_{n}$. Fix an $n \in \mathbb{N}_{0}$. By induction, we show that

$$
\left(x_{n}, x_{n+k}\right) \in R_{n}
$$

for all $k \in \mathbb{N}$. By (3.7), condition (3.8) holds for $k=1$. Assume that (3.8) is satisfied for some $k \in \mathbb{N}$. By $(3.7),\left(x_{n+k}, x_{n+k+1}\right) \in R_{n+k}$. Since $\left(R_{n}\right)_{n=0}^{\infty}$ is descending, we may infer that $\left(x_{n+k}, x_{n+k+1}\right) \in R_{n}$. Hence and by the transitivity, $\left(x_{n}, x_{n+k+1}\right) \in R_{n}$ which completes the induction. Now letting $k$ tend to the infinity in (3.8), we obtain that $\left(x_{n}, x\right) \in R_{n}$ since $R_{n}$ is a closed subset of the product $X \times X$. This completes the proof of Theorem 1.1(iii). Finally, (1.1) is an immediate consequence of the fact that the mapping $F$ is an $\alpha$-contraction.

Corollary 3.2. ET is equivalent to $\mathrm{BCP}^{*}$, the restriction of the $\mathrm{BCP}$ to the class of nonArchimedean bounded metric spaces.

Proof. By Theorem 3.1, the assumptions of ET and BCP* are equivalent. Thus we need here only to verify the suitable property of a sequence of successive approximations. That ET implies BCP* follows from the fact that, under the assumptions of BCP*, if $\left(R_{n}\right)_{n=0}^{\infty}$ is defined by (3.6), then by ET, $F$ has a unique fixed point $x_{*}$ and $\left(F^{n} x, x_{*}\right) \in R_{n}$, that is, $d\left(F^{n} x, x_{*}\right) \leq \alpha^{n} \delta(X)$; in particular, $F^{n} x \rightarrow x_{*}$. We show implication BCP* $\Rightarrow$ ET. Under the assumptions of ET, if $d$ is defined by (3.1), then by BCP* $F$ has a unique fixed point $x_{*}$. Moreover, given $n \in \mathbb{N}_{0}$ and $x \in X$,

$$
d\left(F^{n} x, x_{*}\right)=d\left(F^{n} x, F^{n} x_{*}\right) \leq \alpha^{n} d\left(x, x_{*}\right) \leq \alpha^{n} .
$$

Hence $p\left(F^{n} x, x_{*}\right) \geq n$ which implies that $\left(F^{n} x, x_{*}\right) \in R_{n}$.

Corollary 3.3. Under the assumptions of ET, the intersection $\bigcap_{n \in \mathbb{N}} F^{n}(X)$ is a singleton. Hence a mapping $F$ is not surjective unless $X$ is a singleton. As a consequence, the contraction principle cannot be derived from ET.

Proof. By Theorem 3.1, a mapping $F$ is a Banach contraction with respect to some bounded and complete metric. Then the diameters of sets $F^{n}(X)$ tend to 0 and $F$ has a fixed point $x_{*}$ which implies that $\bigcap_{n \in \mathbb{N}} F^{n}(X)=\left\{x_{*}\right\}$. The second statement is obvious. The last statement follows from the fact that there exist surjective Banach contractions; for such mappings, ET is not applicable.

\section{An extension of Eilenberg's theorem}

The letter $\mathbb{Z}$ denotes the set of all integers.

THEOREM 4.1. Let $X$ be an abstract set and $\left(R_{n}\right)_{n \in \mathbb{Z}}$ a sequence of reflexive and symmetric relations in $X$ such that

(i) given $n \in \mathbb{Z}$, if $(x, y) \in R_{n}$ and $(y, z) \in R_{n}$, then $(x, z) \in R_{n-1}$;

(ii) $\bigcup_{n \in \mathbb{Z}} R_{n}=X \times X$, and $\cdots \supseteq R_{-1} \supseteq R_{0} \supseteq R_{1} \supseteq \cdots$;

(iii) $\bigcap_{n \in \mathbb{Z}} R_{n}=\Delta$; 
(iv) given a sequence $\left(x_{n}\right)_{n=1}^{\infty}$ such that $\left(x_{n}, x_{n+1}\right) \in R_{n}$ for all $n \in \mathbb{N}$, there is an $x \in X$ such that $\left(x_{n}, x\right) \in R_{n-1}$ for all $n \in \mathbb{N}$.

If $F$ is a self-map of $X$ such that given $n \in \mathbb{Z}$ and $x, y \in X$, condition (1.1) is satisfied, then $F$ has a unique fixed point $x_{*}$, and given $x \in X$, there is a $k \in \mathbb{N}$ such that $\left(F^{k+n} x, x_{*}\right) \in R_{n}$ for all $n \in \mathbb{N}$.

Proof. By (ii), given $x \in X$, there is a $p \in \mathbb{Z}$ such that $p<0$ and $(x, F x) \in R_{p}$. Then by (1.1), $\left(F^{-p} x, F^{-p+1} x\right) \in R_{0}$. Denote $y:=F^{-p} x$. Again by (1.1), we get that $\left(F^{n} y, F^{n+1} y\right) \in$ $R_{n}$ for all $n \in \mathbb{N}$, so by (iv), $\left(F^{n} y, x_{*}\right) \in R_{n-1}$ for some $x_{*} \in X$ and for all $n \in \mathbb{N}$. By (1.1), $\left(F^{n+1} y, F x_{*}\right) \in R_{n}$. Since $\left(x_{*}, F^{n+1} y\right) \in R_{n}$, we may infer from (i) that $\left(x_{*}, F x_{*}\right) \in R_{n-1}$. Thus by (iii),

$$
\left(x_{*}, F x_{*}\right) \in \bigcap_{n \in \mathbb{N}_{0}} R_{n}=\bigcap_{n \in \mathbb{Z}} R_{n}=\Delta,
$$

so $x_{*}$ is a fixed point of $F$. A similar argument as in the proof of Theorem 1.1 shows that $x_{*}$ is a unique fixed point. Moreover, $\left(F^{n+1} y, x_{*}\right) \in R_{n}$, that is, $\left(F^{k+n} x, x_{*}\right) \in R_{n}$ with $k:=1-p$.

It is easily seen that ET is subsumed by Theorem 4.1. In particular, condition (i) is weaker than the transitivity of all $R_{n}$. In view of Corollary 3.3, the following result shows the advantage of Theorem 4.1 over Theorem 1.1.

Proposition 4.2. Theorem 4.1 implies the contraction principle.

Proof. Under the assumptions of the contraction principle, define

$$
R_{n}:=\left\{(x, y) \in X \times X: d(x, y) \leq \frac{1}{2^{n}}\right\} \quad \forall n \in \mathbb{Z} .
$$

The triangle inequality implies that Theorem 4.1(i) holds. It is clear that Theorem 4.1(ii) and (iii) are satisfied. To verify Theorem 4.1 (iv), assume that $\left(x_{n}, x_{n+1}\right) \in R_{n}$ for all $n \in \mathbb{N}$, that is, $d\left(x_{n}, x_{n+1}\right) \leq 1 / 2^{n}$. Then $\left(x_{n}\right)_{n=1}^{\infty}$ is a Cauchy sequence, hence convergent to some $x \in X$. Since

$$
d\left(x_{n}, x_{n+k}\right) \leq \sum_{i=n}^{\infty} d\left(x_{i}, x_{i+1}\right) \leq \sum_{i=n}^{\infty} \frac{1}{2^{i}}=\frac{1}{2^{n-1}},
$$

we may infer, letting $k$ tend to the infinity, that $d\left(x_{n}, x\right) \leq 1 / 2^{n-1}$, that is, $\left(x_{n}, x\right) \in R_{n-1}$. Thus Theorem 4.1(iv) holds. Assume that $F: X \mapsto X$ is a Banach contraction. Then there is an $m \in \mathbb{N}$ such that $F^{m}$ is a $1 / 2$-contraction. Denote $G:=F^{m}$. Clearly, given $n \in \mathbb{Z}$, $(x, y) \in R_{n}$ implies that $(G x, G y) \in R_{n+1}$. By Theorem 4.1, $G$ has a unique fixed point $x_{*}$, and given $x \in X$, there is a $k \in \mathbb{N}$ such that $\left(G^{k+n} x, x_{*}\right) \in R_{n}$, that is, $d\left(G^{k+n} x, x_{*}\right) \leq 1 / 2^{n}$ for all $n \in \mathbb{N}$. Hence $G^{n} x \rightarrow x_{*}$. A well-known trick with an iterate of $F$ is to infer that also $F^{n} x \rightarrow x_{*}$ and $x_{*}=F x_{*}$.

Remark 4.3. A variant of ET given by Rus [11] (cf. Remark 2.1) implies the contraction principle for bounded metric spaces (see [11, Theorem 9]). However, the assumptions of his result also force that a mapping $F$ need not be surjective unless $X$ is a singleton. This 
can also be shown without a metric argument. Suppose that $F(X)=X$. Then $F^{n}(X)=X$ for all $n \in \mathbb{N}$. By (1.1),

$$
X \times X=F^{n}(X) \times F^{n}(X) \subseteq R_{n} .
$$

Hence by (1.1), we get that $X \times X=\Delta$, that is, $X$ is a singleton. Thus Rus' theorem cannot be applied if $F$ is a surjective Banach contraction on an unbounded metric space.

We close the paper with the following question.

Question 4.4. Is it possible to find such an extension of ET which would be equivalent to the contraction principle?

\section{References}

[1] J. de Groot, Non-Archimedean metrics in topology, Proc. Amer. Math. Soc. 7 (1956), 948-953.

[2] J. Dugundji and A. Granas, Fixed Point Theory. I, Monografie Matematyczne, vol. 61, PWNPolish Scientific Publishers, Warsaw, 1982.

[3] R. Engelking, General Topology, PWN-Polish Scientific Publishers, Warsaw, 1977.

[4] W. Grudziński, On the discrete Banach principle, Zeszyty Nauk. Politech. Łódz. Mat. (1994), no. 26, 81-88.

[5] J. Jachymski, Equivalent conditions and the Meir-Keeler type theorems, J. Math. Anal. Appl. 194 (1995), no. 1, 293-303.

[6] _ Equivalence of some contractivity properties over metrical structures, Proc. Amer. Math. Soc. 125 (1997), no. 8, 2327-2335.

[7] _ A short proof of the converse to the contraction principle and some related results, Topol. Methods Nonlinear Anal. 15 (2000), no. 1, 179-186.

[8] General solutions of two functional inequalities and converses to contraction theorems, Bull. Polish Acad. Sci. Math. 51 (2003), no. 2, 147-156.

[9] C. Petalas and T. Vidalis, A fixed point theorem in non-Archimedean vector spaces, Proc. Amer. Math. Soc. 118 (1993), no. 3, 819-821.

[10] S. Prieß-Crampe, Der Banachsche Fixpunktsatz für ultrametrische Räume [The Banach fixed point theorem for ultrametric spaces], Results Math. 18 (1990), no. 1-2, 178-186 (German).

[11] I. A. Rus, Discrete fixed point theorems, Studia Univ. Babeş-Bolyai Math. 33 (1988), no. 3, 6164.

Jacek Jachymski: Institute of Mathematics, Technical University of Łódź, Żwirki 36, 90-924 Łódź, Poland

E-mail address: jachym@mail.p.lodz.pl 